\title{
Genotyping-by-Sequencing-Based Genetic Analysis of African Rice Cultivars and Association Mapping of Blast Resistance Genes Against Magnaporthe oryzae Populations in Africa
}

\author{
Emmanuel M. Mgonja, Chan Ho Park, Houxiang Kang, Elias G. Balimponya, Stephen Opiyo, Maria Bellizzi, \\ Samuel K. Mutiga, Felix Rotich, Veena Devi Ganeshan, Robert Mabagala, Clay Sneller, Jim Correll, Bo Zhou, \\ Nicholas J. Talbot, Thomas K. Mitchell, and Guo-Liang Wang ${ }^{\dagger}$
}

\begin{abstract}
First, second, fifth, sixth, ninth, fifteenth, and sixteenth authors: Department of Plant Pathology, The Ohio State University, Columbus; fourth and eleventh authors: Department of Horticulture and Crop science, The Ohio State University, Columbus; third author: State Key Laboratory for Biology of Plant Diseases and Insect Pests, Institute of Plant Protection, Chinese Academy of Agricultural Sciences, Beijing, China; tenth author: Department of Crop Science and Production, Sokoine University of Agriculture, Morogoro, Tanzania; seventh, eighth, and twelfth author: Department of Plant Pathology, University of Arkansas, Fayetteville; seventh author: Biosciences Eastern and Central AfricaInternational Livestock Research Institute (BecA-ILRI) Hub, ILRI Complex, Nairobi, Kenya; thirteenth author: International Rice Research Institute, Los Banos, Philippines; and fourteenth author, School of Biosciences, University of Exeter, UK.
\end{abstract}

Accepted for publication 15 May 2017.

\begin{abstract}
Understanding the genetic diversity of rice germplasm is important for the sustainable use of genetic materials in rice breeding and production. Africa is rich in rice genetic resources that can be utilized to boost rice productivity on the continent. A major constraint to rice production in Africa is rice blast, caused by the hemibiotrophic fungal pathogen Magnaporthe oryzae. In this report, we present the results of a genotyping-by-sequencing (GBS)-based diversity analysis of 190 African rice cultivars and an association mapping of blast resistance $(\mathrm{R})$ genes and quantitative trait loci (QTLs). The 190 African cultivars were clustered into three groups based on the $184 \mathrm{~K}$ single nucleotide polymorphisms

generated by GBS. We inoculated the rice cultivars with six African $M$. oryzae isolates. Association mapping identified 25 genomic regions associated with blast resistance (RABRs) in the rice genome. Moreover, PCR analysis indicated that $R A B R \_23$ is associated with the Pi-ta gene on chromosome 12. Our study demonstrates that the combination of GBSbased genetic diversity population analysis and association mapping is effective in identifying rice blast $\mathrm{R}$ genes/QTLs that contribute to resistance against African populations of $M$. oryzae. The identified markers linked to the RABRs and 14 highly resistant cultivars in this study will be useful for rice breeding in Africa.
\end{abstract}

Rice consumption in Africa and especially in urban areas of the continent has dramatically increased. The current estimated per capita consumption of rice in Africa is $23.3 \mathrm{~kg}$ per annum, which is double the rate of the early 1970s (FAO 2012; Muthayya et al. 2014). Despite this increase in rice consumption, rice production in Africa is facing many biotic and abiotic constraints such as drought, low soil fertility, soil salinity, pests, and diseases. To increase pest and disease resistance and to improve other important agronomic traits, researchers are using molecular breeding and biotechnology in rice breeding programs (Stewart and Ow 2008). The development of sustainable rice production based on these new approaches relies on a detailed understanding and proper management of rice genetic resources and diversity (Guimaraes 2002). Africa has a wealth of rice genetic resources that can be used to expand the genetic base of the rice varieties widely grown across the continent and to develop varieties that are well adapted to abiotic and biotic stresses.

Among the 26 recognized species of rice (Oryza spp.) (USDA National Genetic Resources Program 2012), two are cultivated in Africa: O. sativa and O. glaberrima. While O. glaberrima is cultivated only in West Africa, O. sativa is cultivated where ever

†Corresponding author: G.-L. Wang; E-mail: wang.620@osu.edu

First, second, and third authors contributed equally to this work.

*The $\boldsymbol{e}$-Xtra logo stands for "electronic extra" and indicates that nine supplementary tables and five supplementary figures are published online.

(c) 2017 The American Phytopathological Society rice is grown on the continent. Although yield and genetic diversity are generally lower for $O$. glaberrima than for $O$. sativa, O. glaberrima is better adapted than $O$. sativa to extreme conditions and is tolerant or resistant to high soil salinity, iron toxicity, pests, and diseases (Linares 2002). For these reasons, $O$. glaberrima is a valuable tool for $O$. sativa improvement through interspecific hybridization (Orjuela et al. 2014).

The molecular diversity of African rice cultivars has been analyzed in several studies. Using 93 simple sequence repeat (SSR) markers, Semon et al. (2005) analyzed the population structure of 198 O. glaberrima cultivars from West Africa and identified five genetically diverse groups. The first group contained floating cultivars that originated in the upper delta of the Niger River. The second group contained the nonfloating (lowland) cultivars that are cultivated in Nigeria and that may have originated from areas along the Niger River. The third group contained upland cultivars originating in Liberia. The fourth and fifth groups shared ancestry with two subspecies of $O$. sativa (indica and japonica), indicating that some $O$. glaberrima accessions represent admixtures (Semon 2005). Two separate studies used SSR markers on both $O$. glaberrima and $O$. sativa cultivars from West Africa and obtained similar findings (Barry et al. 2007; Dramé et al. 2013). Another extensive analysis of African rice genetic diversity was done by Orjuela et al. (2014). In their study, which used global genotyping, 279 O. glaberrima cultivars were genotyped into two major groups with 235 single nucleotide polymorphism (SNP) markers. The two populations could not be linked to any currently phenotyped trait such as grain shape, pericarp color, and panicle structure. Misclassifications in O. glaberrima were also identified. 
These findings show that the African cultivars of both $O$. glaberrima and $O$. sativa have a rich diversity that can be further explored.

Next generation sequencing technologies are currently being used for genome sequencing projects, and a large number of SNPs can be obtained at a very low price. These SNPs can be used to study population diversity, to identify genes, and to construct haplotype maps for genome-wide association study (GWAS) (Metzker 2010). Recently, the simple and highly multiplexed genotyping-by-sequencing (GBS) approach has been widely used for diverse organisms (Elshire et al. 2011; Orjuela et al. 2014). GBS is a simple, low cost, highly multiplexed system for constructing reduced representation libraries for the next-generation sequencing platform, which generates large numbers of SNPs. It has become a unique tool for genomics-assisted breeding in many plant species (Beissinger et al. 2013; Poland and Rife 2012).

GWAS uses sequence polymorphisms in natural populations breeding populations or germplasm collections and linkage disequilibrium (LD)-based association to quickly map target genes in a large collection of diverse genotypes. It overcomes the shortcomings of traditional gene mapping using biparental crosses and efficiently maps with high resolution of the loci that are associated with the trait across the whole genome (Abdurakhmonov and Abdukarimov 2008). GWAS can be used to dissect complex traits in crops and to identify multiple genes associated with those traits (Huang et al. 2010; Jia et al. 2013; Sneller et al. 2009).

Rice blast disease, caused by the fungus Magnaporthe oryzae (Bourett and Howard 1990), is a major constraint for rice production in Africa. The use of resistant varieties is the most effective and economic way to manage blast disease (Savary et al. 2012). Africa has rich genetic resources for rice that can be used to identify new resistance genes against $M$. oryzae. Although monogenic gene-forgene resistance provides complete resistance against specific races of $M$. oryzae, this type of resistance is easily broken in the field. Relative to complete resistance, partial resistance controlled by multiple small-effect genes or quantitative trait loci (QTLs) is more durable even under high pathogen population pressure (Mundt 2014). Thus, the breeding of rice blast resistance in elite cultivars requires the use of rice germplasm that contains both major and minor genes. In our previous study (Mgonja et al. 2016), we used 162 cultivars of the rice diversity panel 1 (RDP1); these cultivars were from many countries and were genotyped with 44K SNPs. We inoculated the 162 cultivars with eight $M$. oryzae isolates from four East Africa countries. GWAS was then used to identify genomic regions associated with blast resistance (RABRs) in the rice genome. Seven of these RABRs were linked to known resistance gene loci, and 24 were new. Some are strongly associated with resistance and defense genes encoding NBS-LRR, defense-related proteins, transcription factors, and receptor-like protein kinases.

In this study, we used 190 African rice cultivars from AfricaRice and the Tanzania Ministry of Agriculture. These cultivars were genotyped by GBS and generated 184K SNPs that were used for diversity analysis and association mapping of rice blast $\mathrm{R}$ genes/ QTLs. The 190 African rice cultivars were inoculated with six rice blast isolates from Africa. Phylogenetic analysis using the 184K SNPs led to the identification of three major population groups and the detection of the misclassification of some cultivars. Association mapping with the SNPs and blast inoculation data revealed the presence of $25 \mathrm{RABR}$ in the rice genome. PCR analysis indicated that a major $R A B R$ on chromosome 12 was highly linked with resistance to four of the $M$. oryzae isolates. Our study demonstrates the usefulness of GBS in diversity analysis and in association mapping in rice and has identified 14 highly resistant cultivars to all six isolates and SNP markers linked to RABRs for rice blast breeding in Africa.

\section{MATERIALS AND METHODS}

Plant and fungal materials. The 190 O. glaberrima, O. sativa, and NERICA (New Rice for Africa, hybrids of $O$. glaberrima and
O. sativa) cultivars used in this study were obtained from AfricaRice in Benin and the Tanzania Ministry of Agriculture (Katrin Agricultural Research Institute). These cultivars were collected from all countries where rice is grown in Africa and represent the geographical distributions and ecologies of the cultivated rice species in Africa (Supplementary Table S1). The seeds of these cultivars were increased in a greenhouse at The Ohio State University in the summer of 2013. Dehusked seeds were placed in 15-ml conical tubes and surface sterilized in $70 \%$ ethanol for $1 \mathrm{~min}$ and then in $40 \%$ Clorox for $30 \mathrm{~min}$. The seeds were then washed with sterilized water and placed in Petri dishes containing 1/2 Murashige and Skoog (MS) medium for germination. The $1 / 2 \mathrm{MS}$ medium was prepared by adding $2.2 \mathrm{~g}$ of MS salt, $30 \mathrm{~g}$ of sucrose, and $2.2 \mathrm{~g}$ of Phytagel in 1 liter of distilled water. The $\mathrm{pH}$ was adjusted to $\mathrm{pH} 5.6$ with $1 \mathrm{M} \mathrm{HCl}$ and $\mathrm{KOH}$. Five days after germination, the plantlets were transplanted into sterilized soil in small pots in a growth chamber. The fungal spores were prepared and the plants were spray inoculated as previously described (Mgonja et al. 2016; Park et al. 2012). The six $M$. oryzae isolates used in this study were collected from four African countries: TZ-01 and TZ-12 were from Tanzania, UG-05 and UG-11 were from Uganda, KE-37 was from Kenya, and BF-07 was from Burkina Faso (Mgonja et al. 2016).

Evaluation of rice blast resistance. The blast resistance of the 190 cultivars was evaluated as previously described (Mgonja et al. 2016). In brief, disease was scored 6 days after inoculation using a 0 to 9 blast scale (Kang et al. 2016), in which 0 indicates no blast symptoms (highly resistant) and 9 indicates severe blast symptoms (highly susceptible) (Zhu et al. 2012). The disease inoculation experiment was replicated two times under the same conditions. Whenever results were inconsistent, a third repetition was conducted to obtain results consistent with one of the two previous inoculations.

DNA extraction and GBS. For GBS, genomic DNA was extracted from leaf tissues of a single plant of each of the 190 cultivars using the Qiagen DNeasy DNA extraction kit as per the manufacturer's protocol. The DNA concentration of each sample was quantified using a NanoDrop ND-8000 spectrophotometer. About $30 \mu \mathrm{l}$ of DNA for each cultivar was sent in two 96-well plates to the Institute of Biotechnology at Cornell University where GBS was done using a 96-plex ApeKI GBS protocol. For SNP calling, individual libraries (i.e., cultivars) were identified by their unique names. The reads were then aligned to the genome sequences the $O$. glaberrima and $O$. sativa, respectively, using the BurrowsWheeler transform (BWA) (Li and Durbin 2009). The resulting BAM alignment files were sorted to facilitate the variant calling step. SNPs variants were identified using samtools mpileup program with the following parameters -D 100 (maximum read depth of 100 to call a SNP) (Li et al. 2009). To verify the variants, samtools bcftools view program with the following parameters "-d8 -D100000 -w 2 -Q $20-10.00001-21 \mathrm{e}-100$ - 2 2" were used. Where (-d8 is minimum read depth of 8; -D100,000 is the maximum read depth of 100,000; -w $2=$ SNP within bp around a gap to filter of 2 ; -Q $20=$ minimum mapping quality for SNPs of $20 ;-10.00001=\min P$ value for strand bias of $0.00001 ;-21 \mathrm{e}-100=\min P$ value for baseQ bias of $1 \mathrm{e}-100$; and -p 2 = print filtered variants of 2 .

GBS-based diversity. We used 184K SNP markers generated from GBS to assess the genetic diversity of the 190 African rice cultivars. Genetic distances between the cultivars was calculated using TASSEL (Bradbury et al. 2007), and a phylogenetic tree was constructed using GGTREE R package (Yu et al. 2017).

Association analysis. The phenotypic data (blast disease scores of the 190 African rice cultivars) and the 184K SNP dataset generated by GBS were used to perform GWAS as previously described by Zhao et al. (2011). We used a mixed linear model (MLM) (Bradbury et al. 2007) and Tassel 5.0 software (http://www. maizegenetics.net/tassel/) to run the analysis. To increase the computational power of the MLM, we conducted a compressed MLM, which takes into account the kinship (K) matrix and population structure (Q) matrices (Henderson 1975; Lipka et al. 2012). We 
used the TASSEL results as input files to combine multiple Manhattan plots into an integrated plot (a combined Manhattan plot) using our own Perl scripts based on PERL (Christiansen et al. 2012) and its SVG module (scalable vector graphics). The threshold used for calling candidate QTLs is LOD $\geq 4.0$. We used the SNP with the highest LOD value in each RABR.

PCR analysis. To confirm the GBS-based diversity results, we conducted PCR analysis using rice subspecies-specific primers (Supplementary Table S2) (Bimpong et al. 2011; Hirano et al. 1994). Thirty-eight of the 190 African rice cultivars were randomly chosen. Three $O$. glaberrima accessions obtained from the National Small Grains Collection in Idaho, USA (Tog 5603, Tog 6126, and Tog 84117) and the japonica cultivar Nipponbare (NPB) and indica cultivar CO-39 (included in the 190 cultivars) were included as controls (Supplementary Table S3). Amplifications were performed in a $25-\mu \mathrm{l}$ reaction mixture containing $50 \mathrm{ng} / \mu \mathrm{l}$ genomic DNA, $1 \mathrm{X}$ reaction buffer (Promega), $0.25 \mathrm{mM}$ dNTPs, $0.2 \mu \mathrm{M}$ random primer, $2.5 \mu \mathrm{M} \mathrm{MgCl}_{2}$, and 1 unit of Taq polymerase. The PCR amplification and product electrophoresis procedures were previously described (Chen et al. 2009).

To investigate the association of the $P i$-ta gene with the observed resistance reactions, PCR analysis of RABR_23 was done using Pi-taspecific primers. DNA from 10 resistant cultivars and 10 susceptible cultivars were used for the analysis (Supplementary Table S4). The PCR conditions were as follows: predenaturation for $3 \mathrm{~min}$ at $95^{\circ} \mathrm{C}$; followed by 33 cycles for $30 \mathrm{~s}$ at $95^{\circ} \mathrm{C}$ for denaturation, $60 \mathrm{~s}$ at $58^{\circ} \mathrm{C}$ for primer annealing, and $60 \mathrm{~s}$ for extension at $72^{\circ} \mathrm{C}$; and a final extension step for $5 \mathrm{~min}$ at $72^{\circ} \mathrm{C}$.

\section{RESULTS}

GBS-based diversity analysis of African rice cultivars. We generated 184K SNP markers from the GBS data and used these markers to assess the genetic diversity of the 190 African rice cultivars. The analyses showed that the 190 African cultivars fall into three groups, two large ones and a small one that consists of only 20 cultivars (Fig. 1). Surprisingly, the groupings were independent of cultivar origin and presumed subspecies. The first group (Grp I) contained 116 cultivars. Among them, 85 cultivars originated from Tanzania (TZLRs) and 31 from AfricaRice germplasm (ARs) cultivars. Most cultivars in this group were classified as $O$. sativa although some were classified as $O$. glaberrima. Moreover, most NERICA varieties ( $O$. glaberrima and $O$. sativa hybrids) were clustered together in Grp I. Grp II contained 53 cultivars including 47 cultivars from AfricaRice and six cultivars from Tanzania. Among the 20 cultivars in Grp III, 13 were from AfricaRice, and seven were from Tanzania. The results indicate that most of the cultivars with the $O$. glaberrima background are included in Grp I.

To confirm the above results, we performed PCR analysis using the primer pair M14 that is specific to the $O$. glaberrima genome

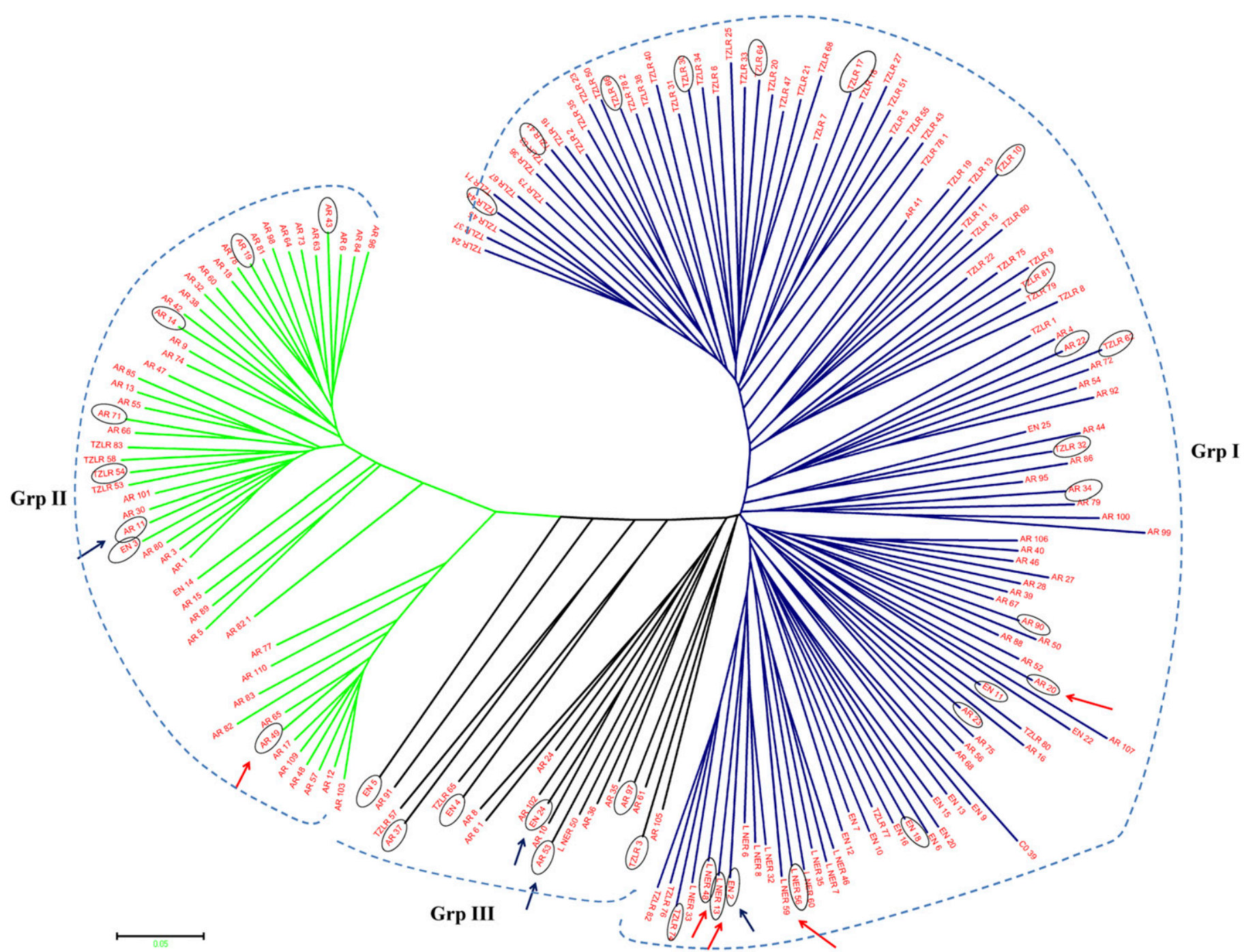

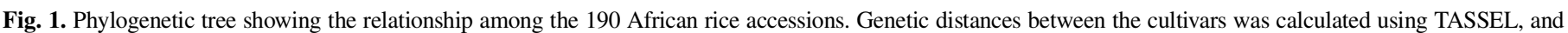

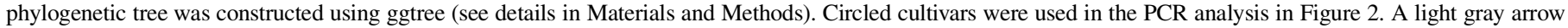

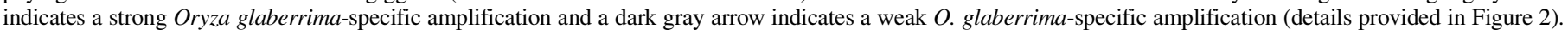


(Bimpong et al. 2011). DNA from 38 cultivars randomly chosen from the 190 cultivars was used for PCR assay. The assay revealed that the three $O$. glaberrima accessions (numbers 38 to 40) from the USDA Small Grains Collection and five cultivars (number 8, 17, 18, 20, and 23) had a strong $O$. glaberrima-specific band (Fig. 2, first panel). Except AR49 in Grp II, the other four cultivars with a strong O. glaberrima-specific band were included in Grp I. Among the five cultivars, only numbers 17,18 , and 20 were labeled as "interspecific" cultivars. In addition, another four cultivars had a weak $O$. glaberrimaspecific band plus an $O$. sativa-specific band. Two cultivars with the weak bands of both $O$. sativa and O. glaberrima were included in Grp I, one was in Grp I and another one in Grp II. Except number 3 was labeled as "interspecific," other three were $O$. sativa cultivars. These results demonstrate that some of the rice cultivars in the AfricaRice and Tanzania germplasm centers may be mislabeled or cross-contaminated.

Blast disease reactions of the 190 cultivars. The original inoculation data of 190 cultivars to six isolates are shown in Supplementary Table S5 and the distributions of the blast disease scores (mean of two replications) are shown in Supplementary Figure S1. The mean score against the isolates ranged from 3.4 (for isolate BF-07) to 4.9 (for isolate UG-05). The percentages of cultivars that were resistant and susceptible to each isolate and the mean disease scores are shown in Supplementary Table S6. Based on the disease reactions of the 190 African cultivars, 14 were highly resistant (disease score $\leq 2.0$ ) to all six isolates Supplementary Table S7. Cluster analysis of the disease reactions of the rice cultivars indicated that the six isolates were different from each other and did not form clusters based on their origin (Supplementary Fig. S2).
Only the two isolates from Uganda (UG-05 and UG-11) were clustered together $(\approx 93 \%$ similarity), and these two isolates were related to TZ-01 from Tanzania. Interestingly, an isolate from Tanzania (TZ-12) was clustered together with an isolate from Burkina Faso (BF-07).

Identification of rice QTLs associated with resistance to six isolates. Using the SNP and disease phenotypic data sets, we conducted GWAS and identified 25 nonredundant RABRs (LOD $\geq$ $4.0)$ in the rice genome against the six $M$. oryzae isolates in the African rice cultivars. $R A B R \_3, R A B R \_20, R A B R \_22$, and $R A B R \_23$ are located in the regions with the previously mapped $\mathrm{R}$ genes $P i$-sh, $P i-y(t), P i-6$, and Pi-ta, respectively. Two of these four RABRs ( $R A B R \_3$ and $R A B R \_20$ ) were also identified in our previous study using 162 RDP1 cultivars (Mgonja et al. 2016). Three of the 25 RABRs ( $R A B R \_20, R A B R \_22$, and $\left.R A B R \_23\right)$ were identified previously (Kang et al. 2016). Only one RABR $(R A B R-20)$ of our 25 RABRs was reported by Wang et al. (2014). The remaining 19 RABRs are novel candidate QTLs (Fig. 3). Supplementary Table S8 summarizes the information on chromosome locations and candidate genes for all 25 RABRs. No RABR was associated with all six isolates. $R A B R \_7$ and $R A B R \_8$ were associated with resistance to all of the isolates from East Africa except isolate BF-07 from Burkina Faso. Thirteen RABRs were effective against at least two isolates. However, there was no association between shared RABRs and country of origin for the isolates.

The 25 RABRs were distributed across the 12 rice chromosomes (Supplementary Figs. S3, S4, and S5). The highest number of RABRs per chromosome was four (observed on chromosomes 1, 2,

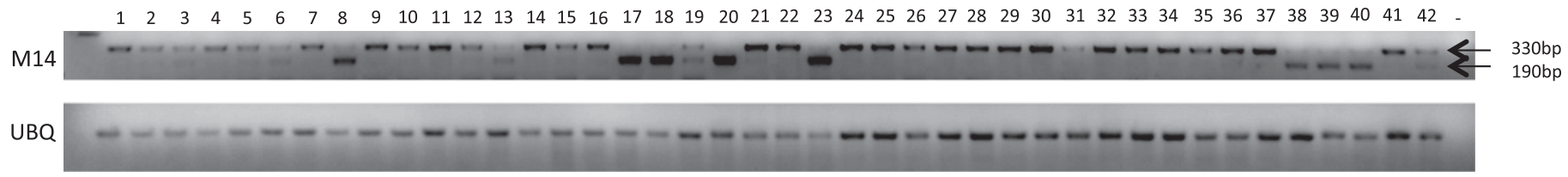

Fig. 2. Gel images of the PCR analysis of 38 cultivars with the Oryza glaberrima-specific primer pair M14. The names and origins of the 38 cultivars are listed in Supplementary Table S4. Three $O$. glaberrima accessions (numbers 38 to 40), japonica cultivar Nipponbare (number 41 ), and indica CO-39 (number 42 ) were included as controls. The primer sequences are listed in Supplementary Table S2. PCR conditions are described in Materials and Methods.

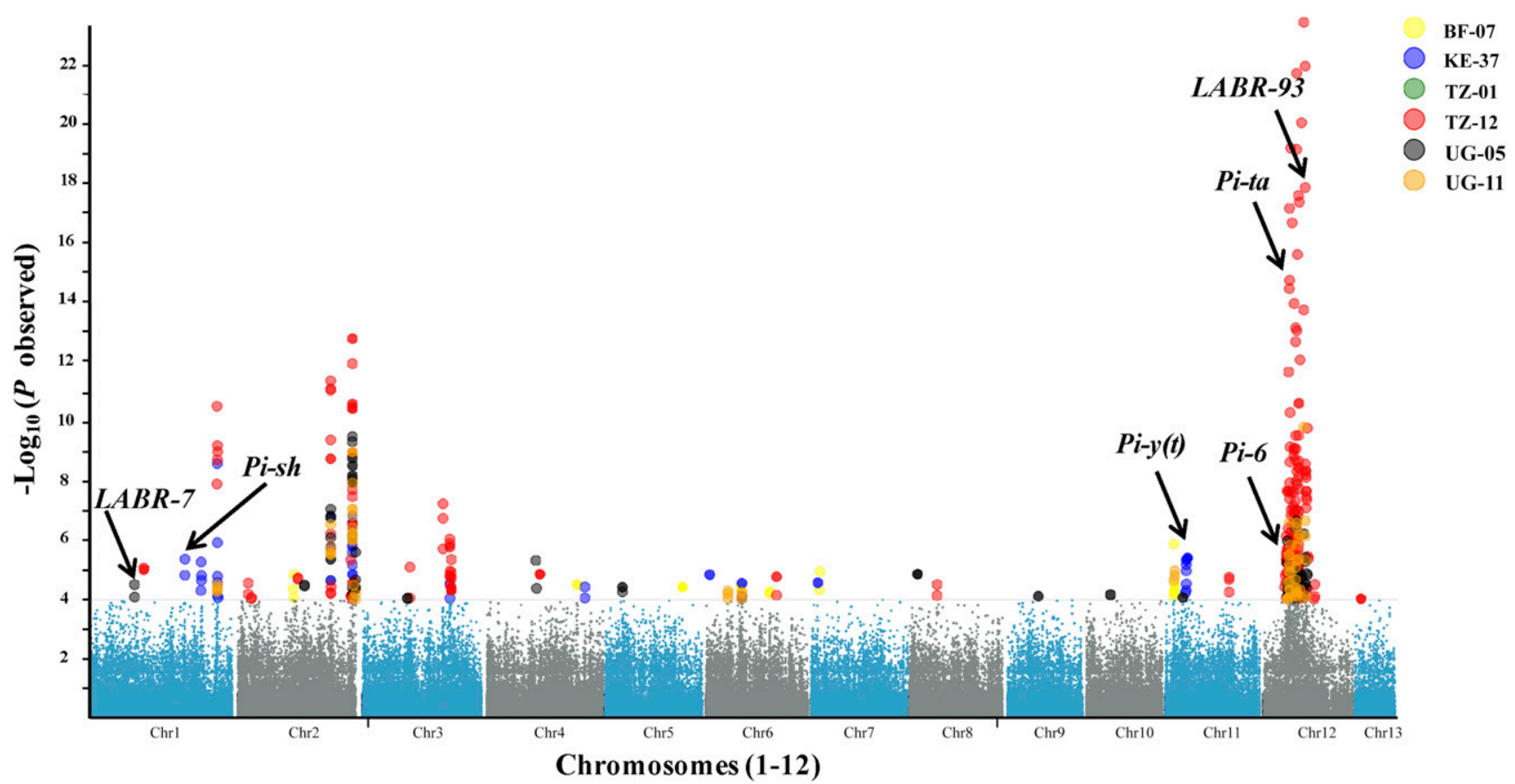

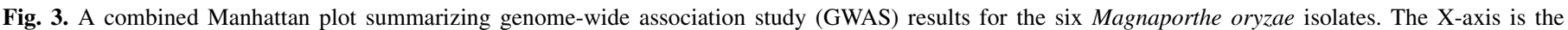

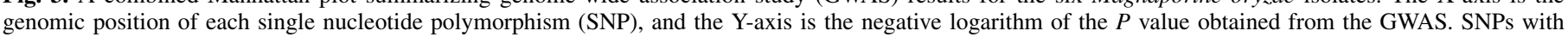

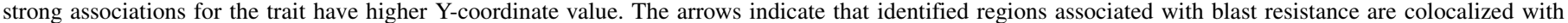
previously mapped or cloned $\mathrm{R}$ gene regions. 


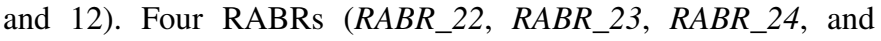
$R A B R 25)$ were clustered together on chromosome 12 within about a $4.3 \mathrm{Mb}$ region and were strongly associated with resistance to four isolates (UG-05, UG-11, TZ-12, and BF-07). RABR_23 had the highest LOD scores in response to isolate TZ-12 (LOD -20) (Fig. 4). We further identified candidate genes associated with the 25 RABRs by exploring the Nipponbare genomic sequence (MSU V7.0) using gene ontology (GO) terms. The identified $25 \mathrm{R}$ or defense-related candidate genes in the 25 RABRs are listed in Supplementary Table S8. The 25 candidate genes included genes encoding NBS-LRR proteins, receptor-like protein kinases, phosphorylation-related proteins, transcription factors, ubiquitinationrelated proteins, and DNA-binding proteins. Confirmation of the functions of these genes is needed using genetic and molecular methods.

$\boldsymbol{R} A B R \_23$ is linked to the $\mathrm{R}$ gene $\boldsymbol{P} \boldsymbol{i}$-ta. The strong association of $R A B R \_23$ with resistance to four isolates prompted us to perform a detailed analysis of this genomic region on chromosome 12 . Sequence analysis showed that $R A B R \_23$ is closely linked to $P i$ - $t a$, which encodes an NBS-LRR domain R protein (Bryan et al. 2000) and provides wide spectrum resistance against different isolates of $M$. oryzae in India and other rice-growing countries in Asia (Ramkumar et al. 2014). Using three Pi-ta-specific primer pairs (Pita5, 577, and 801), as shown in Supplementary Table S9 (Hayashi et al. 2006), we amplified the Pi-ta homologous gene in 10 resistant and 10 susceptible cultivars that were used in our pathogenicity assay. Using the Pita5 $\mathrm{R}+\mathrm{F}$ primer pair, we were able to amplify a fragment related to $P i$-ta in 8 of 10 resistant cultivars and in 2 of 10 susceptible cultivars (Fig. 5). A similar result was obtained when another primer pair, Pita801R+F, was used in the
PCR analysis. To confirm the association of $R A B R \_23$ with $P i$-ta, we inoculated the $P i$-ta-containing cultivar Tadukan with the three isolates (TZ-12, UG-05, and UG-11) that showed strong association with $R A B R \_23$ (Jia et al. 2004). The inoculation showed that Tadukan was resistant to all three isolates, confirming that $R A B R \_23$ is associated with $P i$-ta and contributes significantly to the resistance reactions in some of the 190 African rice cultivars. These results suggest that $R A B R \_23$ might be a new allele of the $P i$ ta gene and might be useful in rice blast resistance breeding programs in Africa.

Highly resistant rice varieties are enriched for resistance alleles. Next, we compared the SNP genotypes across each of the 25 RABRs in the 14 rice cultivars that were highly resistant to all six isolates and the 14 rice cultivars that were highly susceptible to all isolates. Interestingly, the 14 highly resistant cultivars had about $86 \%$ resistance-associated SNP genotypes in the 25 loci when the most significant SNP in each region was selected to represent the genotype of cultivars. In contrast, the 14 highly susceptible cultivars had only $20 \%$ resistance-associated SNP genotypes in the 25 loci (Fig. 6). These results indicated that at the blast-associated QTL loci identified in this study, the 14 highly resistant rice varieties are enriched for resistance alleles while the 14 high susceptible varieties are enriched for susceptibility alleles.

\section{DISCUSSION}

Sustainable rice production depends on the preservation and wise use of genetic materials. With the increased intensification of rice cropping and the rapid evolution of $M$. oryzae, populations of the
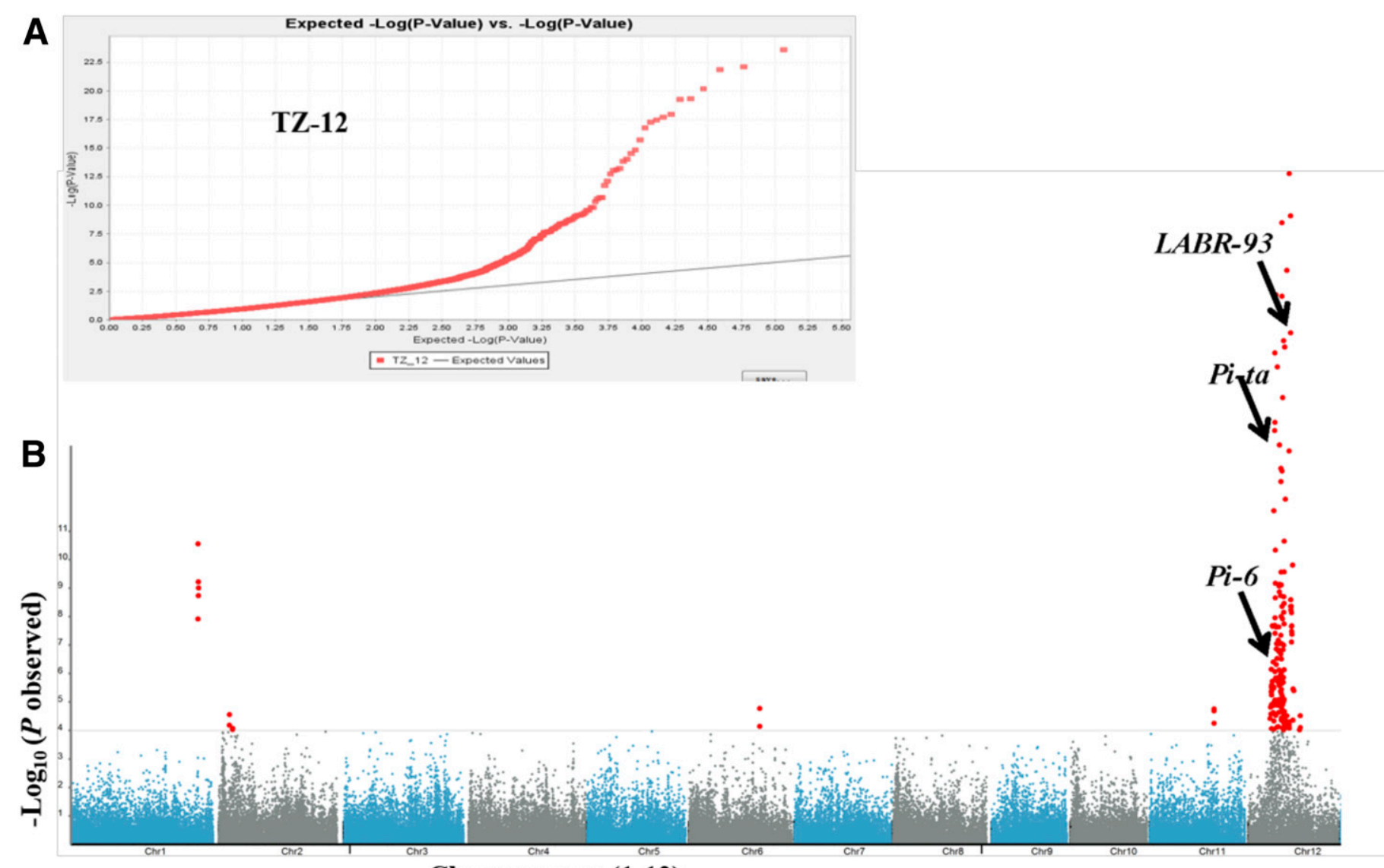

Expected Value

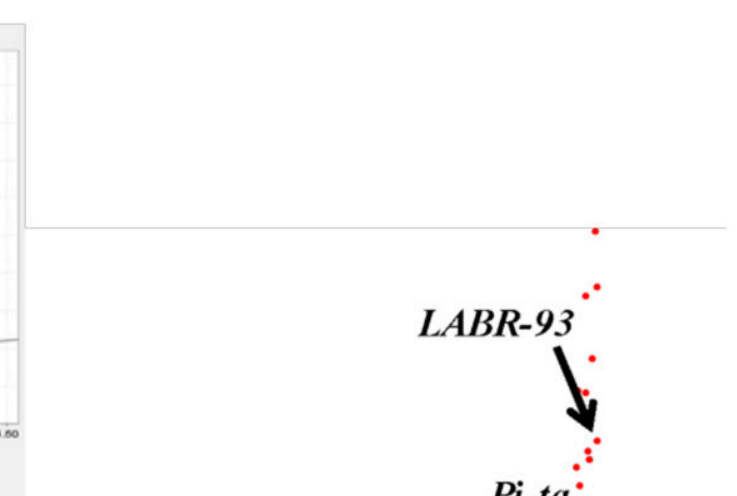

B
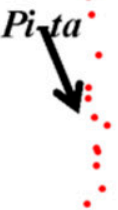

$\cdot$

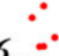

Chromosomes (1-12)

Fig. 4. Genome-wide association analysis for Tanzanian Magnaporthe oryzae isolate TZ-12. A, Quantile-quantile plots that shows fitness of the genome-wide association study (GWAS) analysis model for the isolate. The X-axis corresponds to the expected values of negative logarithm of $P$ and the Y-axis corresponds to the observed values of negative logarithm of $P$ value. B, Manhattan plots that summarize GWAS results. The X-axis is the genomic position of each single nucleotide polymorphism (SNP), and the Y-axis is the negative logarithm of the $P$ value obtained from the GWAS model. SNPs with strong associations for the trait (blast disease resistance) have higher Y-coordinate value. 
pathogen that can break monogenic resistance can develop within a few years in the field (Valent and Khang 2010). Therefore, we need to investigate rice genetic pools to identify additional $\mathrm{R}$ genes and QTLs that can be used for resistance breeding programs in changing environments. Africa has a wealth of rice genetic resources that can be exploited for increasing the resistance or tolerance of rice cultivars to biotic and abiotic challenges across the continent (Sanni et al. 2013). In the current study, we used a diverse rice germplasm collection from AfricaRice in West Africa and the Ministry of Agriculture in Tanzania in East Africa. The cultivars represent a wide geographical diversity and adaptation as they were collected from many rice-growing countries on the continent. Using the GBS technique, we genotyped all of the cultivars and obtained $184 \mathrm{~K}$ SNPs. We also inoculated these cultivars with six M. oryzae isolates collected from West and East African countries. With the SNP and disease score datasets, we performed an association mapping and identified 25 RABRs. Among them, 19 are new candidate R/QTLs that have not been reported before. Our results demonstrate the power of combining local germplasm collections with a GBS-based association mapping approach to identify new genes/QTLs that provide resistance against diverse $M$. oryzae isolates in Africa.

A number of molecular diversity analyses of African rice cultivars have revealed that African rice is diverse and often misclassified as $O$. sativa or O. glaberrima (Orjuela et al. 2014; Semon et al. 2005). The 190 African rice cultivars in the current study included those with $O$. sativa, O. glaberrima, and interspecific hybrid (NERICAs) backgrounds. The phylogenetic analysis with the $184 \mathrm{~K}$ SNPs showed that these cultivars can be classified into three major groups. However, the grouping was independent of origin/ecology or background recorded in the germplasm. This lack of a relationship between grouping and origin or background could be due to misclassification of some cultivars, to the creation of admixtures in the handling of the seeds, or to the natural hybridization between $O$. sativa and $O$. glaberrima (Barry et al. 2007; Dramé et al. 2013). Previous studies reported the occurrence of natural hybrids between $O$. sativa and $O$. glaberrima (Barry et al. 2007; Semon et al. 2005), but a subsequent study demonstrated that these are $O$. sativa and not interspecific hybrids (Garris et al. 2005). However, the putative hybrids are not classified as $O$. sativa indica or $O$. sativa japonica and might have been introduced a long time ago in Africa from Asia (Orjuela et al. 2014). In our PCR analysis using an O. glaberrima-primer pair (Fig. 2), only five cultivars contained the O. glaberrima-specific sequence. These results show the possibility that some of the African rice cultivars are misclassified in terms of name and origin. Studies with highly polymorphic and abundant SNP markers are needed to properly classify the African germplasm. Proper classification would increase the value of the germplasm as a genetic stock and enable sustainable and easy exploitation of the genetic pool.

Rice blast is a major disease in many rice-growing areas of Africa (Onaga et al. 2015). Lack of highly resistant rice cultivars, climate change, increasing use of fertilizers, and rice intensification make the disease even more damaging. Identification of new resistance genes in the African rice germplasm is urgently needed. Using the 190 African rice cultivars and six M. oryzae isolates from Tanzania, Uganda, Kenya, and Burkina Faso, we identified 25 RABRs. Six of the 25 RABRs are colocalized with blast resistance loci reported in other three previous studies (Kang et al. 2016; Mgonja et al. 2016; Wang et al. 2014). Three of these six RABRs (RABR_20, RABR_22, and $R A B R \_23$ ) closely relate to three RABRs reported by Kang et al. (2016). $R A B R-20$ is similar to one of those reported previously (Wang et al. 2014). The other two out of six RABRs were identified in our previous gene mapping study using 162 varieties from RDP1 (Mgonja et al. 2016). Nineteen of the twenty-five RABRs are new, suggesting that African rice populations may contain many blast resistance loci that differ from those in non-African populations.

Many association mapping studies have shown that resistance to $M$. oryzae isolates is controlled by multiple loci with $\mathrm{R}$ genes and minor QTLs (Liu et al. 2013). We found that 5 of the 25 RABRs had LOD scores of $\geq 7.0$, which implies that these loci may have relative

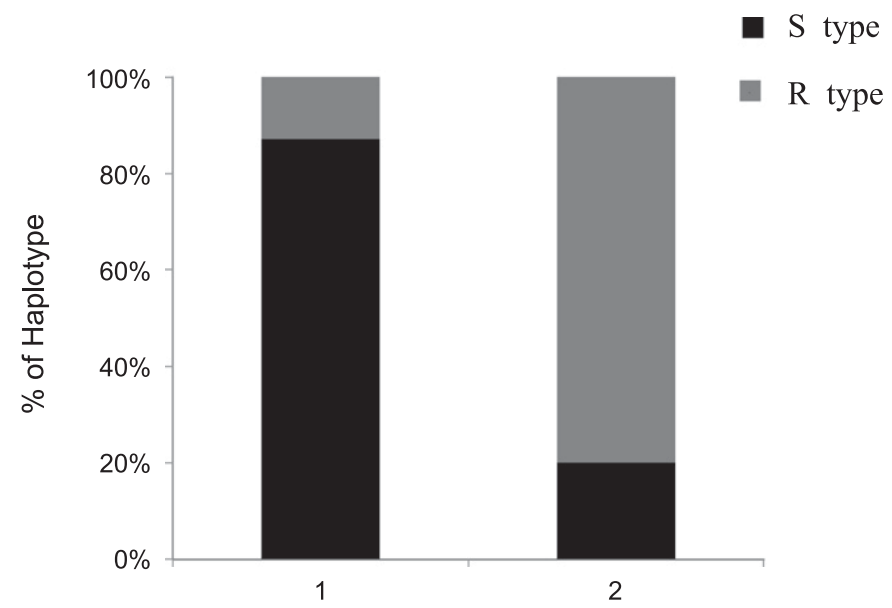

1: The 14 highly susceptible cultivars

2: The 14 highly resistant cultivars

Fig. 6. Highly resistant rice varieties are enriched for resistance alleles. The frequency of the single nucleotide polymorphism genotypes across each of the 25 regions associated with blast resistance were compared in the 14 rice highly resistant and the 14 highly susceptible cultivars. R: Resistant; S: Susceptible.
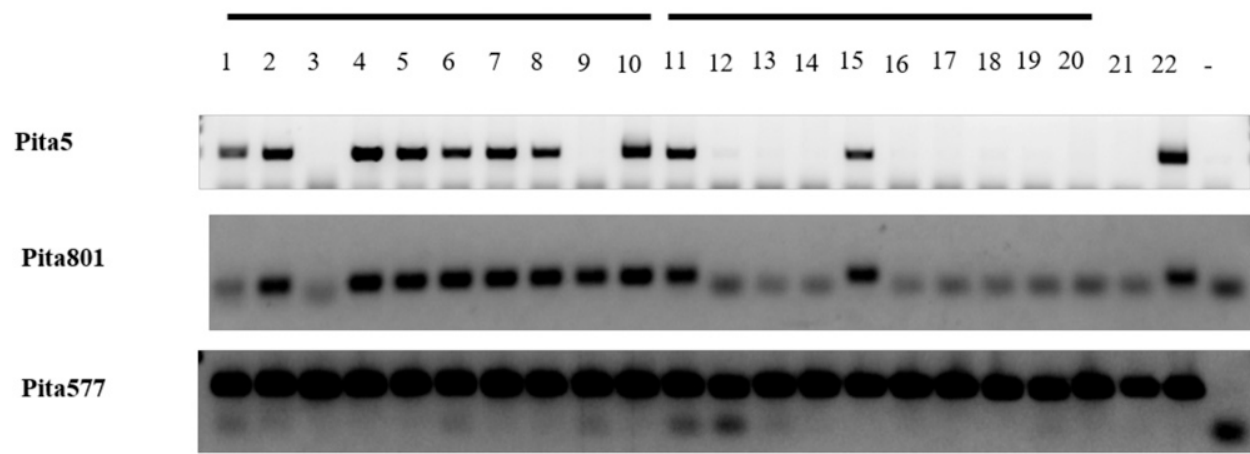

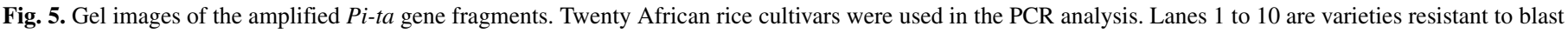

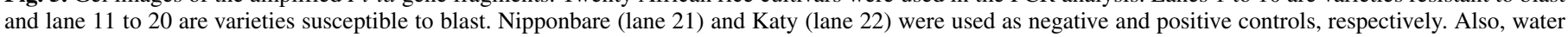

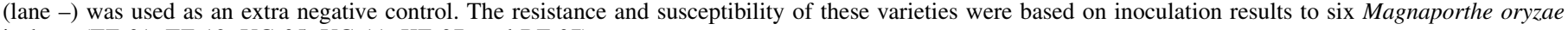
isolates (TZ-01, TZ-12, UG-05, UG-11, KE-37, and BF-07). 
big contributions to resistance compared with the rest of the 20 RABRs with LOD scores of $\leq 7$. This illustrates that both African rice germplasm and $M$. oryzae populations are complex and that the resistance phenotypes are controlled by relative large QTLs and minor QTLs. Because QTLs are important for the durability of rice blast resistance in the field, their identification will facilitate their fine-mapping and use as markers in rice breeding programs in Africa.

$R A B R \_23$ on chromosome 12 is strongly associated with blast resistance. We found that this locus is linked to the Pi-ta gene. PCR amplifications of the RABR_23-related candidate gene in 10 resistant and 10 susceptible cultivars confirmed the association of the locus with the resistance phenotype. However, we found that the candidate gene was amplified by $P i$-ta-specific primers in two susceptible cultivars and was not amplified in two resistant cultivars. This indicates that $P i-t a$ is not the only locus contributing to resistance, i.e., other R genes/QTLs contribute to resistance in these cultivars. Nevertheless, RABR_23 was associated with resistance to three isolates from East Africa (UG-05, UG-11, and TZ-12) and one isolate from West Africa (BF-07), suggesting its usefulness as a candidate $\mathrm{R}$ gene for managing rice blast in East and West Africa.

Based on the pathogenicity results, 14 cultivars were highly resistant (disease score of $\leq 2.0$ ) to all six $M$. oryzae isolates. These cultivars have about $86 \%$ resistance-associated SNP genotypes in the 25 loci and may contain multiple R genes and/or QTLs that are effective against African $M$. oryzae populations. Because these cultivars are locally adapted and have good agronomic traits, they will be easier to use than resistant cultivars from other regions in rice breeding programs in Africa.

\section{ACKNOWLEDGMENTS}

This project was supported in part by grants from the SCPRID program of BBSRC and BM Gates Foundation (BB/J012157/1), the Innovative Agricultural Research Initiative of the USAID, the Borlaug LEAP fellowship, and the Natural Science Foundation of China (NSFC, number 31461143019). The African rice seeds were obtained from AfricaRice in Benin and Katrin Agricultural Research Institute (ARI-Katrin) in Tanzania.

\section{LITERATURE CITED}

Abdurakhmonov, I. Y., and Abdukarimov, A. 2008. Application of association mapping to understanding the genetic diversity of plant germplasm resources. Int. J. Plant Genomics 2008:574927.

Barry, M. B., Pham, J. L., Noyer, J. L., Billot, C., Courtois, B., and Ahmadi, N. 2007. Genetic diversity of the two cultivated rice species (O. sativa \& O. glaberrima) in Maritime Guinea. Evidence for interspecific recombination. Euphytica 154:127-137.

Beissinger, T. M., Hirsch, C. N., Sekhon, R. S., Foerster, J. M., Johnson, J. M., Muttoni, G., Vaillancourt, B., Buell, C. R., Kaeppler, S. M., and de Leon, N. 2013. Marker density and read depth for genotyping populations using genotyping-by-sequencing. Genetics 193:1073-81.

Bimpong, I. K., Chin, J. H., Ramos, R., and Koh, H. J. 2011. Application of subspecies-specific marker system identified from Oryza sativa to Oryza glaberrima accessions and Oryza sativa $\times$ Oryza glaberrima $\mathrm{F} 1$ interspecific progenies. Int. J. Genet. Mol. Biol. 3:7-24.

Bourett, T. M., and Howard, R. J. 1990. In vitro development of penetration structures in the rice blast fungus Magnaporthe grisea. Can. J. Bot. 68: 329-342.

Bradbury, P. J., Zhang, Z., Kroon, D. E., Casstevens, T. M., Ramdoss, Y., and Buckler, E. S. 2007. TASSEL: Software for association mapping of complex traits in diverse samples. Bioinformatics 23:2633-2635.

Bryan, G. T., Wu, K.-S., Farrall, L., Jia, Y., Hershey, H. P., McAdams, S. A., Faulk, K. N., Donaldson, G. K., Tarchini, R., and Valent, B. 2000. A single amino acid difference distinguishes resistant and susceptible alleles of the rice blast resistance gene Pi-ta. Plant Cell 12:2033-2045.

Chen, S. B., Songkumarn, P., Liu, J. L., and Wang, G. L. 2009. A versatile zero background T-vector system for gene cloning and functional genomics. Plant Physiol. 150:1111-21.

Christiansen, T., Foy, B. D., and Wall, L., and Orwant, J. 2012. Programming Perl-Unmatched Power for Text Processing and Scripting. 4th ed. O'Reilly Media, Sebastopol, CA.
Dramé, K. N., Sanchez, I., Gregorio, G., and Ndjiondjop, M. N. 2013. Suitability of a selected set of simple sequence repeats (SSR) markers for multiplexing and rapid molecular characterization of African rice (Oryza glaberrima Steud.). Afr. J. Biotechnol. 10:6675-6685.

Elshire, R. J., Glaubitz, J. C., Sun, Q., Poland, J. A., Kawamoto, K., Buckler, E. S., and Mitchell, S. E. 2011. A robust, simple genotyping-by-sequencing (GBS) approach for high diversity species. PLoS One 6:e19379.

FAO. 2012. The State of Food Insecurity in the World 2012: Economic growth is necessary but not sufficient to accelerate reduction of hunger and malnutrition. FAO, Rome. http://www.fao.org/docrep/016/i3027e/i3027e.pdf

Garris, A. J., Tai, T. H., Coburn, J., Kresovich, S., and McCouch, S. 2005. Genetic structure and diversity in Oryza sativa L. Genetics 169:1631-1638.

Guimaraes, E. P. 2002. Genetic diversity of rice production in Brazil. Pages 11-35 in: Genetic Diversity in Rice Production, Case Studies from Brazil, India and Nigeria. Food and Agriculture Organization of the United Nations. V. N. Nguyen, ed. FAO, Rome, Italy.

Hayashi, K., Yoshida, H., and Ashikawa, I. 2006. Development of PCR-based allele-specific and InDel marker sets for nine rice blast resistance genes. Theor. Appl. Genet. 113:251-60.

Henderson, C. R. 1975. Best linear unbiased estimation and prediction under a selection model. Biometrics 31:423-447.

Hirano, H. Y., Mochizuki, K., Umeda, M., Ohtsubo, H., Ohtsubo, E., and Sano, Y. 1994. Retrotransposition of a plant SINE into the wx locus during evolution of rice. J. Mol. Evol. 38:132-7.

Huang, X., Wei, X., Sang, T., Zhao, Q., Feng, Q., Zhao, Y., Li, C., Zhu, C., Lu, T., Zhang, Z., Li, M., Fan, D., Guo, Y., Wang, A., Wang, L., Deng, L., Li, W., Weng, Q., Liu, K., Huang, T., Zhou, T., Jing, Y., Li, W., Lin, Z., Buckler, E. S., Qian, Q., Zhang, Q., Li, J., and Han, B. 2010. Genome-wide association studies of 14 agronomic traits in rice landraces. Nat. Genet. 42: 961-967.

Jia, G., Huang, X., Zhi, H., Zhao, Y., Zhao, Q., Li, W., Chai, Y., Yang, L., Liu, K., and Lu, H. 2013. A haplotype map of genomic variations and genomewide association studies of agronomic traits in foxtail millet (Setaria italica). Nat. Genet. 45:957-961.

Jia, Y., Wang, Z., Fjellstrom, R. G., Moldenhauer, K. A., Azam, M. A., Correll, J., Lee, F. N., Xia, Y., and Rutger, J. N. 2004. Rice Pi-ta gene confers resistance to the major pathotypes of the rice blast fungus in the United States. Phytopathology 94:296-301.

Kang, H., Wang, Y., Peng, S., Zhang, Y., Xiao, Y., Wang, D., Qu, S., Li, Z., Yan, S., Wang, Z., Liu, W., Ning, Y., Korniliev, P., Leung, H., Mezey, J., McCouch, S. R., and Wang, G. L. 2016. Dissection of the genetic architecture of rice resistance to the blast fungus Magnaporthe oryzae. Mol. Plant Pathol. 17:959-72.

Li, H., and Durbin, R. 2009. Fast and accurate short read alignment with Burrows-Wheeler transform. Bioinformatics 25:1754-60.

Li, H., Handsaker, B., Wysoker, A., Fennell, T., Ruan, J., Homer, N., Marth, G., Abecasis, G., and Durbin, R. 2009. The sequence alignment/Map format and SAMtools. Bioinformatics 25:2078-2079.

Linares, O. F. 2002. African rice (Oryza glaberrima): History and future potential. Proc. Natl. Acad. Sci. 99:16360-16365.

Lipka, A. E., Tian, F., Wang, Q., Peiffer, J., Li, M., Bradbury, P. J., Gore, M. A., Buckler, E. S., and Zhang, Z. 2012. GAPIT: Genome association and prediction integrated tool. Bioinformatics 28:2397-2399.

Liu, Y., Liu, B., Zhu, X., Yang, J., Bordeos, A., Wang, G., Leach, J. E., and Leung, H. 2013. Fine-mapping and molecular marker development for Pi56 (t), a NBS-LRR gene conferring broad-spectrum resistance to Magnaporthe oryzae in rice. Theor. Appl. Genet. 126:985-98.

Metzker, M. L. 2010. Sequencing technologies-The next generation. Nat. Rev. Genet. 11:31-46.

Mgonja, E. M., Balimponya, G., Kang, H., Bellizzi, M., Park, C. H., Li, Y., Mabagala, R., Sneller, C., Correll, J., Opiyo, S., Talbot, N. J., Mitchell, T., and Wang, G. L. 2016. Genome-wide association mapping of rice resistance genes against Magnaporthe oryzae isolates from four African countries. Phytopathology 106:1359-1365.

Mundt, C. C. 2014. Durable resistance: A key to sustainable management of pathogens and pests. Infect. Genet. Evol. 27:446-55.

Muthayya, S., Sugimoto, J. D., Montgomery, S., and Maberly, G. F. 2014. An overview of global rice production, supply, trade, and consumption. Ann. N.Y. Acad. Sci. 1324:7-14.

Onaga, G., Wydra, K., Koopmann, B., Sere, Y., and von Tiedemann, A. 2015. Population structure, pathogenicity, and mating type distribution of Magnaporthe oryzae isolates from East Africa. Phytopathology 105:1137-1145.

Orjuela, J., Sabot, F., Chéron, S., Vigouroux, Y., Adam, H., Chrestin, H., Sanni, K., Lorieux, M., and Ghesquière, A. 2014. An extensive analysis of the African rice genetic diversity through a global genotyping. Theor. Appl. Genet. 127:2211-2223.

Park, C. H., Chen, S., Shirsekar, G., Zhou, B., Khang, C. H., Songkumarn, P., Afzal, A. J., Ning, Y., Wang, R., Bellizzi, M., Valent, B., and Wang, G. L. 2012. The Magnaporthe oryzae effector AvrPiz-t targets the RING E3 
ubiquitin ligase APIP6 to suppress pathogen-associated molecular patterntriggered immunity in rice. Plant Cell 24:4748-62.

Poland, J. A., and Rife, T. W. 2012. Genotyping-by-sequencing for plant breeding and genetics. Plant Genome 5:92-102.

Ramkumar, G., Madhav, M. S., Rama Devi, S. J. S., Manimaran, P., Mohan, K. M., Balachandran, S. M., Neeraja, C. N., Sundaram, R. M., Viraktamath, B. C., and Prasad, M. S. 2014. Nucleotide diversity of Pita, a major blast resistance gene and identification of its minimal promoter. Gene 546:250-256.

Sanni, K. A., Tia, D. D., Ojo, D. K., Ogunbayo, A. S., Sikirou, M., and Hamilton, N. R. S. 2013. Diversity of Rice and Related Wild Species in Africa. Page 87 in: Realizing Africa's Rice Promise. CABI Publishing.

Savary, S., Horgan, F., Willocquet, L., and Heong, K. 2012. A review of principles for sustainable pest management in rice. Crop Prot. 32:54-63.

Semon, M., Nielsen, R., Jones, M. P., and McCouch, S. R. 2005. The population structure of African cultivated rice Oryza glaberrima (Steud.) evidence for elevated levels of linkage disequilibrium caused by admixture with $O$. sativa and ecological adaptation. Genetics 169:1639-1647.

Sneller, C. H., Mather, D. E., and Crepieux, S. 2009. Analytical approaches and population types for finding and utilizing QTL in complex plant populations. Crop Sci. 49:363-380.
Stewart, C. N., and Ow, D. W. 2008. The future of plant biotechnology. Pages 357-369 in: Plant Biotechnology and Genetics: Principles, Techniques, and Applications. John Wiley \& Sons, Hoboken, NJ.

USDA National Genetic Resources Program. 2012. Germplasm Resources Information Network-(GRIN) [Online Database]. National Germplasm Resources Laboratory, Beltsville, MD. https://www.ars-grin.gov/

Valent, B., and Khang, C. H. 2010. Recent advances in rice blast effector research. Curr. Opin. Plant Biol. 13:434-441.

Wang, C., Yang, Y., Yuan, X., Xu, Q., Feng, Y., Yu, H., Wang, Y., and Wei, X. 2014. Genome-wide association study of blast resistance in indica rice. BMC Plant Biol. 14:311.

Yu, G. C., Smith, D. K., Zhu, H. C., Guan, Y., and Lam, T. T. Y. 2017. GGTREE: An $R$ package for visualization and annotation of phylogenetic trees with their covariates and other associated data. Methods Ecol. Evol. 8:28-36.

Zhao, K., Tung, C.-W., Eizenga, G. C., Wright, M. H., Ali, M. L., Price, A. H., Norton, G. J., Islam, M. R., Reynolds, A., Mezey, J., McClung, A. M., Bustamante, C. D., and McCouch, S. R. 2011. Genome-wide association mapping reveals a rich genetic architecture of complex traits in Oryza sativa. Nat. Commun. 2:467.

Zhu, X. Y., Chen, S., Yang, J. Y., Zhou, S. C., Zeng, L. X., and Han, J. L. 2012. The identification of $\mathrm{Pi50}(\mathrm{t})$, a new member of the rice blast resistance Pi2/Pi9 multigene family. Theor. Appl. Genet. 124:1295-1304. 